\title{
Alcohol, tabaco y deterioro cognoscitivo en adultos mexicanos mayores de 65 años
}

\author{
Sara Gloria Aguilar-Navarro, MC, (I) Jorge Reyes-Guerrero, MC, (I) Guilherme Borgues, D en SP.(2)
}

\author{
Aguilar-Navarro SG, Reyes-Guerrero J, Borgues G. \\ Alcohol, tabaco y deterioro cognoscitivo \\ en adultos mexicanos mayores de 65 años. \\ Salud Publica Mex 2007;49 supl 4:S467-S474.
}

\begin{abstract}
Resumen
Objetivo. Conocer la prevalencia del consumo de alcohol y tabaco y su asociación con deterioro cognoscitivo en la población mexicana mayor de 65 años. Material y métodos. Se incluyeron 4872 mayores de 65 años en la muestra del Estudio Nacional sobre Salud y Envejecimiento en México (ENASEM 200I). Se interrogó sobre el consumo de alcohol y tabaco. Para la clasificación de los sujetos con deterioro cognoscitivo, se utilizó la escala total de los diferentes dominios cognoscitivos. Se aplicaron ji cuadrada, Mann Whitney $U$ y regresión logística para encontrar asociaciones. Resultados. La prevalencia de alcoholismo según CAGE fue de $2.8 \%$ y la del consumo de tabaco de 14 por ciento. Factores asociados con el consumo de alcohol: edad 65-69 años $(p<0.001)$, hombres (RR 3.17, $p<0.001)$, nivel alto de educación $(p<0.001)$. Con asociación significativa entre el consumo de alcohol y deterioro cognoscitivo $\left(\chi^{2}=6.59, p<\right.$ 0.01 ). Conclusiones. La prevalencia de consumo de alcohol y tabaco en los adultos mayores mexicanos es similar a lo reportado en otros países; el consumo de alcohol se asoció con el deterioro cognoscitivo.
\end{abstract}

Palabras clave: alcoholismo, tabaquismo, deterioro cognoscitivo, adulto mayor, ENASEM; México
Aguilar-Navarro SG, Reyes-Guerrero J, Borgues G. Cognitive impairment and alcohol and cigarette consumption in Mexican adults older than 65 years. Salud Publica Mex 2007;49 suppl 4:S467-S474.

\begin{abstract}
Objective.To determine the prevalence of the consumption of alcohol and cigarette smoking and their association with cognitive impairment among older Mexican adults. Material and Methods. A total of 4872 people over 65 years of age included in the sample of the National Mexican Health and Aging Study carried out in 200 I were questioned about their consumption of alcohol and cigarette smoking. For the classification of those subjects with cognitive impairment, the total scale of the different cognitive domains was used. The chi-square, Mann-Whitney $U$ test, and logistical regression were used in order to find associations. Results. The prevalence of alcoholism according to CAGE was $2.8 \%$ and the prevalence of the consumption of cigarette smoking was $14 \%$. Factors associated with the consumption of alcohol were:age 65-69 ( $p<0.001)$, men (RR 3.17, $p<0.001)$, and high level of education $(p<0.001)$. The association between the consumption of alcohol and cognitive impairment $(X 2=6.59$, $p<0.0$ I) was statistically significant. Conclusions. The prevalence of consumption of alcohol and cigarette smoking in older Mexican adults are similar to that reported in other countries; the consumption of alcohol and its association with cognitive impairment was significant.
\end{abstract}

Key words: alcohol; smoking; cognitive; older adults; MHAS (Mexican Health and Aging Study); Mexico

(I) Instituto Nacional de Ciencias Médicas y Nutrición Salvador Zubirán. México.

(2) Instituto Nacional de Psiquiatría. México.

Fecha de recibido: 22 de septiembre de 2005 - Fecha de aceptado: 9 de mayo de 2007

Solicitud de sobretiros: Dra. Sara Gloria Aguilar-Navarro, Instituto Nacional de Ciencias Médicas y Nutrición Salvador Zubirán. Vasco de Quiroga I5, colonia Sección XVI. I4000,Tlalpan, México, DF, México.

Correo electrónico: sganajma@yahoo.com.mx 
$\mathbf{E}_{y}^{1}$ abuso en el consumo de sustancias como alcohol y tabaco es un problema de salud pobremente reconocido en el anciano. Los trastornos relacionados con sustancias se dividen en dos grupos: trastornos por consumo de sustancias (dependencia y abuso) y trastornos inducidos por sustancias (intoxicaciones, abstinencia, delirium, demencia persistente y trastornos amnésicos). Según el Manual de diagnóstico y estadístico de los trastornos mentales. Texto revisado (DSM-IV-TR), la dependencia de sustancias se caracteriza por un patrón desadaptativo de consumo de la sustancia que conlleva un deterioro o malestar clínicamente significativo, expresado por tres (o más) de los ítems siguientes en algún momento de un periodo continuado de 12 meses: tolerancia, abstinencia, frecuencia, deseo persistente, empleo de tiempo en la obtención de la sustancia, reducción importante de actividades sociales y persistencia a pesar de los efectos adversos. ${ }^{1}$ Ewing agrega a este listado: el consumo recurrente que da lugar al incumplimiento de las obligaciones; consumo recurrente en situaciones en las que hacerlo es físicamente peligroso; problemas legales y sociales. ${ }^{2}$

Hacer la distinción entre dependencia al alcohol y abuso es difícil. En la bibliografía es frecuente que ambos términos se utilicen indistintamente. Los estudios epidemiológicos revelan una prevalencia de desórdenes en la forma de beber hasta en $3 \%$ en hombres ancianos y $0.46 \%$ en mujeres; sin embargo, en estudios comunitarios se observa que hasta $10 \%$ de los ingresos a las unidades de urgencias en mayores de 65 años se deben al abuso en el consumo de alcohol. Además, se ha encontrado que, debido a la falta de una distinción clara entre alcoholismo y abuso de alcohol, estudios previos han considerado como fuerte consumidor al anciano que ingiere más de dos bebidas diarias. Con esto la prevalencia llega hasta 6 por ciento. Según la Encuesta Nacional de Adicciones en México 2002, 72\% de la población masculina y 42\% de la femenina mayor de 18 años consume alcohol. ${ }^{3-6} \mathrm{El}$ consumo de alcohol en el anciano es menor que en la población adulta joven. Los factores que contribuyen a reducir su consumo en esta etapa de la vida son el mayor efecto psicológico con cantidades menores, los cambios fisiológicos y la pérdida de función social. Sin embargo también se ha observado mayor riesgo de abuso de alcohol en adultos mayores de 70 años que viven solos. ${ }^{7}$ Es por esto que debe fomentarse el estudio del alcoholismo en el adulto mayor al igual que en el joven, planteando un modelo de tres vectores: biológico, psicológico y social. El caso que nos ocupa, el alcoholismo en la vejez, debe tratarse en todos los aspectos en forma más incisiva con el fin de abordar las complicaciones médicas asociadas, ya que su justa valoración es especialmente difícil a causa de la alta comorbilidad (no sólo del SNC, sino también de otros sistemas). Trabajos previos han estudiado en el anciano alcohólico la disfunción cognitiva relacionada con el abuso de alcohol, las desestructuraciones familiares, sintomatología distímica y ansiosa, manifestaciones somáticas y de tipo neurovegetativo. ${ }^{8-10}$ Los ancianos minimizan el impacto negativo del consumo de tabaco en su salud y el potencial beneficio de dejar de fumar.

Existen instrumentos validados para identificar problemas relacionados con el consumo y abuso de alcohol en la población que nos ocupa, como el utilizado en este estudio; el cuestionario CAGE ${ }^{3,4}$ que consta de cuatro preguntas: 1. ¿Alguna vez ha considerado disminuir la cantidad de bebidas alcohólicas que toma? 2. ¿Alguna vez le molestó que la gente lo criticara sobre el hecho de que toma? 3. ¿Se siente Ud. mal o culpable por la forma que toma? 4. ¿Toma alguna bebida alcohólica al levantarse por las mañanas para calmar sus nervios o para deshacerse de la "cruda"? Dos o más preguntas positivas indican que existe una problemática relacionada con el consumo del alcohol; cuatro preguntas positivas indican alcoholismo. Se ha reportado una especificidad de $56-100 \%$ y una sensibilidad de $60-70$ por ciento. Este instrumento discrimina a partir de la historia del consumo de alcohol y se utilizó en este análisis para identificar los casos. Otros instrumentos como Short Michigan Alcoholism Screening Test-Geriatric Version (SMAST-G), ${ }^{4,5}$ con una sensibilidad de $95 \%$ y especificidad de $78 \%$, también ayudan a identificar ancianos con problemas en el consumo de alcohol. Por último, cabe mencionar otro cuestionario ampliamente utilizado en otros países, como el Alcohol Use Disorders Test (AUDIT). ${ }^{4,6,11}$ Los ancianos minimizan el impacto negativo del consumo de tabaco en su salud y el potencial beneficio de dejar de fumar. Reportes en las últimas dos décadas revelan que dejar de fumar es beneficioso en todos los grupos de edad, incluyendo ancianos. Algunos de estos beneficios son: disminución del riesgo de enfermedad cardiaca, enfermedad vascular cerebral y función pulmonar. El consumo de tabaco se considera actualmente un factor de riesgo prevenible de morbilidad y mortalidad prematura en gran parte del mundo; ha sido reconocido como una adicción de inicio en la adolescencia, ya que se ha observado el efecto de los hijos o nietos de fumadores con mayor tendencia al uso y abuso de esta sustancia. La prevalencia en Estados Unidos es de 10.6-15\% en mayores de 65 años; sin embargo, en los últimos años ha habido un descenso principalmente en los hombres, y se ha observado un mayor consumo en personas con menor nivel educativo y estado civil soltero. ${ }^{11-13}$

Se ha reportado que la ingesta leve o moderada de alcohol reduce el riesgo de enfermedad de Alzheimer y demencia vascular. Sin embargo, también se ha obser- 
vado que el uso y abuso crónico de alcohol tiene efecto negativo y puede exacerbar los síntomas de deterioro cognoscitivo y daño cerebral irreversible. ${ }^{11-13}$ Datos provenientes del Estudio Indianápolis, Salud envejecimiento y prevalencia de deterioro cognoscitivo en ancianos, revelaron que el consumo y abuso de alcohol se relaciona con deterioro cognoscitivo, no demencia, con una prevalencia en la comunidad hasta de $1.6 \%$, y en tres casos se encontró una asociación con enfermedad vascular y demencia, por lo que se consideró el abuso de alcohol como un importante factor de riesgo en el estudio de pacientes con algún tipo de deterioro cognoscitivo. ${ }^{14}$ Las alteraciones neurocognoscitivas que se han observado en pacientes con historia de abuso de alcohol y demencia son: problemas visuoespaciales y alteraciones ejecutivas como enlentecimiento del pensamiento, perseveraciones y confabulaciones comunes, indicadores de alteración en las funciones ejecutivas en pacientes que beben alcohol en forma crónica. La alteración de la memoria es amnesia anterógrada, mientras que el lenguaje generalmente se preserva. ${ }^{15-17}$

Contrario a lo que previamente se conocía respecto al efecto protector del tabaco sobre la enfermedad de Alzheimer, han surgido estudios recientes que lo han reconocido como un factor de riesgo para demencia. La exposición crónica al tabaco causa arterioesclerosis e hipertensión subsiguiente. Estos factores, sumados a la agregación plaquetaria aumentada a corto plazo y a la vasoconstricción arterial, explican el riesgo marcadamente elevado de ictus y posiblemente de infartos silentes. En el estudio European Community Concerted Action Epidemiology of Dementia (Eurodem), se evaluó la relación entre fumar y cambios negativos en el Mini-Mental State Examination (MMSE), y se encontró un descenso importante del MMSE en sujetos que fumaban contra sujetos no fumadores $(p<0.0001)$ (Eurodem). ${ }^{18-22}$

El objetivo de este estudio es conocer la prevalencia del consumo y abuso de alcohol y tabaco en la población anciana mexicana y su asociación con deterioro cognoscitivo, ya que en México no hay datos precedentes.

\section{Material y métodos}

De los 17503 sujetos que se seleccionaron, 16861 resultaron elegibles. De éstos, 15186 contaron con entrevistas completas, y 4872 eran mayores de 65 años, mismos que se incluyeron en el estudio de prevalencia. Se excluyeron todos aquellos que no tenían código de identificación, y se obtuvo una muestra de 4862 (99.8\%), con autorreporte completo de consumo de alcohol y tabaco. Para el subanálisis de asociación con deterioro cognoscitivo se incluyeron 2838 sujetos que contaban con evaluación cognoscitiva completa. La muestra se obtuvo a partir del Estudio Nacional sobre Salud y Envejecimiento en México (ENASEM 2001), representativa de los 13 millones de mexicanos nacidos antes de 1951, con dos estratos de residencia: urbano y rural. Se sobremuestrearon seis entidades de las que provienen aproximadamente $40 \%$ de los emigrantes a Estados Unidos, con una tasa de poco menos de 2:1. Se analizaron características sociodemográficas como edad, sexo, estado civil, nivel de educación y situación laboral; se interrogó sobre el antecedente en el consumo de alcohol, consumo actual y número de días de ingesta por semana. El ENASEM nació de la necesidad de conocer el estado de salud y los antecedentes de la mayoría de los inmigrantes a Estados Unidos de América; se trata de un estudio prospectivo de mexicanos nacidos antes de 1951 y de sus cónyuges/compañeros sin importar su edad, cuyos objetivos y diseño están disponibles en línea. ${ }^{23}$ Se aplicó un total de 9806 entrevistas personales o de un sustituto, y se logró entrevistar a 5424 cónyuges / compañeros. El ENASEM examinó el proceso de envejecimiento e impacto de las enfermedades y discapacidades. La encuesta contempla, entre otros aspectos, información acerca el estado funcional, hábitos personales como el consumo de tabaco y alcohol, acceso a los servicios de salud, depresión, dolor, desempeño cognoscitivo; sin embargo, en este rubro es importante señalar que sólo se completó la evaluación cognoscitiva en 2838 casos, debido a que, cuando el sujeto contaba con alguna limitación física (déficit sensorial o emocional) que limitaba su evaluación, el cuestionario podía ser respondido por un familiar. En este estudio sólo se analizaron las evaluaciones cognoscitivas con respuestas realizadas por el propio sujeto. También se incluyeron mediciones antropométricas e información sobre condiciones de vida en la niñez, educación, historia marital, economía y disponibilidad de bienes de consumo duradero. Para estimar la prevalencia de alcoholismo, se utilizó el cuestionario CAGE. Para consumo de tabaco se interrogó sobre antecedentes y tabaquismo actual, número de cigarros y número de años. Para obtener el índice tabáquico (IT) se hizo el siguiente cálculo: (número de cigarrillos $x$ número de años) / 20 . Se incluyeron los sujetos con un IT $\geq 10$.

Para la clasificación de los sujetos con deterioro cognoscitivo, se utilizó una distribución percentilar en los dominios cognoscitivos de atención, memoria primaria, memoria verbal secundaria y praxis de construcción; se analizó la distribución en percentiles de la población, los cuales fueron obtenidos por escalas en orden ascendente de lo más bajo a lo más alto en cada nivel de educación (0, 1-4, 5-9 y 10 o más años). El criterio para clasificar a los pacientes con deterioro cognoscitivo fue por debajo del percentil 10 (deterioro severo). 
Para determinar la presencia o ausencia de síntomas depresivos se utilizó una pregunta que hace énfasis en el estado de ánimo actual, a la cual respondían si se sentían o no deprimidos. Los datos obtenidos de cada entrevista fueron capturados en distintas bases de datos, para distintos rubros, tales como datos demográficos, medidas de salud, funcionalidad, cognición, etcétera. Por medio de estadística descriptiva, ji cuadrada, Mann Whitney U y un análisis de regresión logística, se buscó la relación entre consumo de alcohol, fumar y deterioro cognoscitivo. El formato utilizado para la construcción de la base de datos el y análisis estadístico fue el programa estadístico SPSS, versión 10.

\section{Resultados}

En el grupo que consumía alcohol y el que consumía tabaco, el rango de edad predominante fue de 65-74 años. En ambos grupos predominó el sexo femenino; pero los de mayor consumo fueron del masculino. La mayoría reportó estar casado y predominó la situación laboral desempleado. Tanto los que consumían alcohol como tabaco reportaron mayor independencia para actividades básicas de la vida diaria (ABVD) y actividades instrumentales de la vida diaria (AIVD) (figura 1). Un $58.3 \%$ ( $n=2838$ ) contó con datos completos de evaluación cognitiva (cuadro I). De éstos, 9.8\% (277) presentó deterioro cognoscitivo (cuadro II).

Consumo de alcohol y tabaco

Del total de la muestra, 1688 sujetos $(34.7 \%)$ reportaron consumo de alcohol en el pasado. De éstos, $67.5 \%$ ( $n=$ 1140) continuaban haciéndolo en el presente, con una ingesta de más de cuatro bebidas diarias en los últimos tres meses de hasta 23\% (18\% hombres y $6 \%$ mujeres). La prevalencia de alcoholismo, analizada a partir del cuestionario CAGE con cuatro preguntas positivas, fue de 2.8\% (figura 2). En el consumo de tabaco se observó que 2253 sujetos (46.3\%) de la población mayor de 65 años reportaron consumo de tabaco en el pasado. El consumo activo se presentó en $23 \%$ de la población anciana $(n=688)$, de los cuales $73 \%$ pertenecía al sexo masculino y $27 \%$ al femenino, con un IT mayor de 10 en $14.5 \%$ de los sujetos.

\section{Variables asociadas con el consumo de alcohol y tabaco}

En este análisis se encontró que el grupo de edad entre 65-70 años presentó mayor probabilidad de consumo y abuso de alcohol y de tabaco $(p<0.001),(p=$ 0.08 ) respectivamente. Asimismo, los sujetos del sexo masculino tienen mayor probabilidad de consumo de alcohol $[R R=3.17$ (2.6 a 3.8), $p<0.001]$, situación que no

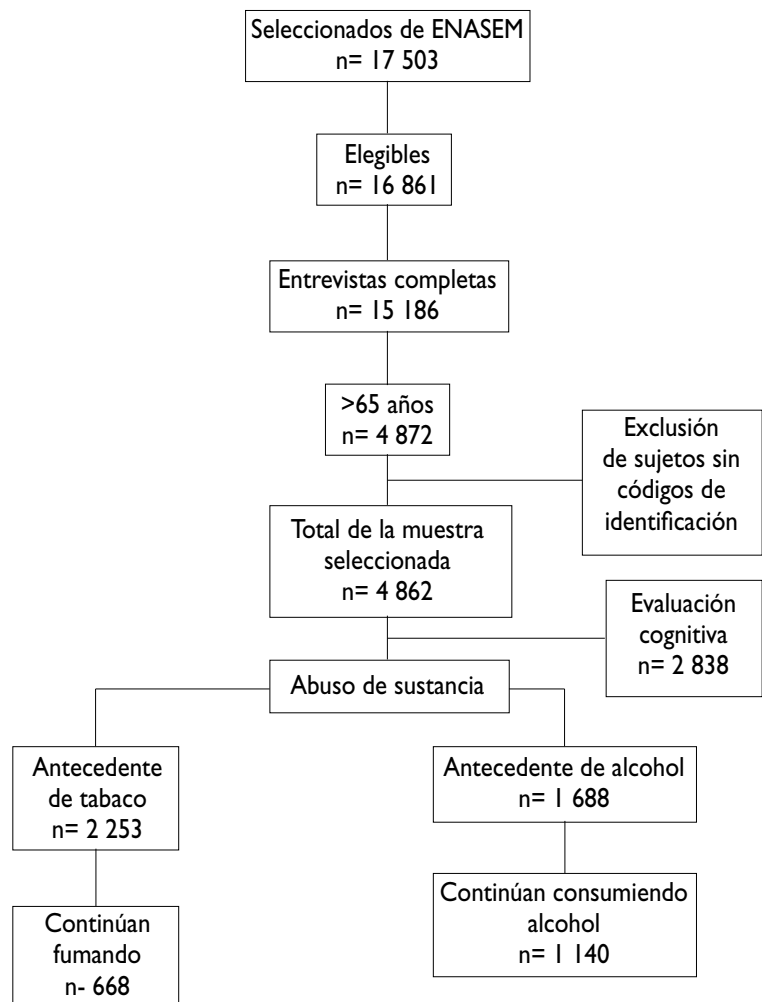

* ENASEM 200I, México, D.F: INEGI

Figura I. Selección de la muestra de estudio

se dio en los sujetos que consumían tabaco. Además, la mayor escolaridad se asoció también con el mayor consumo de alcohol $(p<0.001)$; el desempleo con el mayor consumo de tabaco $(R R=1.21$, IC $0.9-1.23$; $p=$ $0.083) ;$. la dependencia funcional en actividades básicas de la vida diaria con el menor consumo de alcohol y tabaco $(R R=0.7$; IC 0.6-0.9; $p=0.051)$; de igual manera, la dependencia funcional en actividades instrumentales $(R R=0.7, I C$ 0.5-0.9; $p=0.018)$. Al buscar la asociación de síntomas depresivos con abuso de estas sustancias, no se obtuvo un riesgo significativo; por el contrario, se asoció a menor consumo de alcohol y tabaco $(R R=0.80$; IC 0.6-0.9; $p=0.012$ ) (cuadro III).

Asociación de consumo de alcohol y tabaco con deterioro cognoscitivo

En el subanálisis para explorar la asociación con deterioro cognoscitivo, se encontró que los antecedentes de consumo de alcohol y tabaco no muestran asociación con el deterioro cognoscitivo. El consumo actual de tabaco tampoco mostró asociación con el deterioro 


\section{Cuadro I}

Características de los adultos mexicanos mayores de 65 años en estudio ( $=4862$ )

\begin{tabular}{|c|c|c|c|c|c|}
\hline \multirow[b]{2}{*}{ Variable } & \multicolumn{2}{|c|}{ Porcentaje de consumo de tabaco } & \multicolumn{3}{|c|}{ Porcentaje de consumo de alcohol } \\
\hline & $\begin{array}{c}\text { Antecedentes } \\
n=2253\end{array}$ & $\begin{array}{r}\text { Actual } \\
n=688\end{array}$ & $\begin{array}{c}\text { Abstemios } \\
\mathrm{n}=\mathrm{I} 495\end{array}$ & $\begin{array}{c}\text { Antecedentes } \\
\text { n I } 686\end{array}$ & $\begin{array}{c}\text { Actual } \\
n=|| 40\end{array}$ \\
\hline
\end{tabular}

Edad (años)

\begin{tabular}{rrrrrrrr}
$65-74$ & 65 & 64 & 68 & 63 & 63 & 72 \\
\hline $74-85$ & 27 & 29 & 28 & 28 & 29 & 24 \\
\hline$>85$ & 8 & 7 & 4 & 9 & 8 & 4
\end{tabular}

Sexo

\begin{tabular}{lllllll} 
Femenino & 72 & 30 & 27 & 81 & 33 & 27 \\
\hline Masculino & 28 & 70 & 73 & 19 & 67 & 73
\end{tabular}

Educación (años)

\begin{tabular}{crrrrrrr}
$0-6$ & 88 & 88 & 80 & 88 & 90 & 83 \\
\hline $7-12$ & 8 & 8.5 & 17 & 8 & 8 & 10 \\
\hline$>12$ & 4 & 3.5 & 3 & 4 & 2 & 7
\end{tabular}

Estado civil

\begin{tabular}{lrrrrrrr} 
Soltero (a) & 5 & 3 & 3 & 4 & 4 & 3 \\
\hline Casado (a) & 49 & 61 & 64 & 45 & 60 & 63 \\
\hline Divorciado (a) & 6 & 8 & 7 & 7 & 28 & 28
\end{tabular}

Situación de empleo

\begin{tabular}{lllllll} 
Empleado (a) & 29 & 39 & 46 & 24 & 35 & 46 \\
\hline Desempleado (a) & 71 & 61 & 54 & 76 & 65 & 54
\end{tabular}

ABVD

\begin{tabular}{llllllll} 
Funcional & 76 & 76 & 80 & 75 & 73 & 86 \\
\hline Dependiente & 24 & 24 & 20 & 25 & 27 & 14
\end{tabular}

AIVD

\begin{tabular}{lllllll} 
Funcional & 87 & 88 & 94 & 87 & 84 & 91 \\
\hline Dependiente & 13 & 22 & 6 & 13 & 16 & 9 \\
\hline
\end{tabular}

cognoscitivo. Pero uno de los hallazgos más importantes de este análisis fue la asociación entre el consumo actual de alcohol con el deterioro cognoscitivo $\left(\chi^{2}=6.59\right.$, $p=0.01)$; además, a más días de ingesta de alcohol por semana se asoció una mayor probabilidad de deterioro cognoscitivo $\left(\chi^{2}=7, p=0.03\right)$ (cuadro IV).

\section{Conclusiones}

En México pocos estudios han reportado el impacto del consumo y abuso de alcohol en el anciano. Solís y co- laboradores ${ }^{24}$ reportaron en una población general hasta $20.5 \%$ de consumo de alcohol y $3.8 \%$ de prevalencia en síndrome de dependencia al alcohol en ancianos. En Estados Unidos, Adams y colaboradores ${ }^{15}$ reportaron una prevalencia de la problemática relacionada con la ingesta de alcohol en el anciano hasta 15 por ciento. Con otros instrumentos de tamizaje, como el Alcohol Use Disorders Identification Test (AUDIT), ${ }^{28}$ y el AlcoholRelated Problems Survey (ARPS), ${ }^{28}$ se ha reportado una prevalencia de $11 \%$ para síndrome de dependencia al alcohol y hasta de 35\% para problemática relacionada 
Cuadro II

Adultos meXicanos mayores de 65 años con datos

DE COGNICIÓN COMPLETOS

$(\mathrm{N}=2$ 838)

\begin{tabular}{lrc} 
Cognición & $n=2838$ & Porcentaje \\
Mayor percentil 10 & 2561 & 90.2 \\
\hline Menor percentil 10 & 277 & 9.8
\end{tabular}

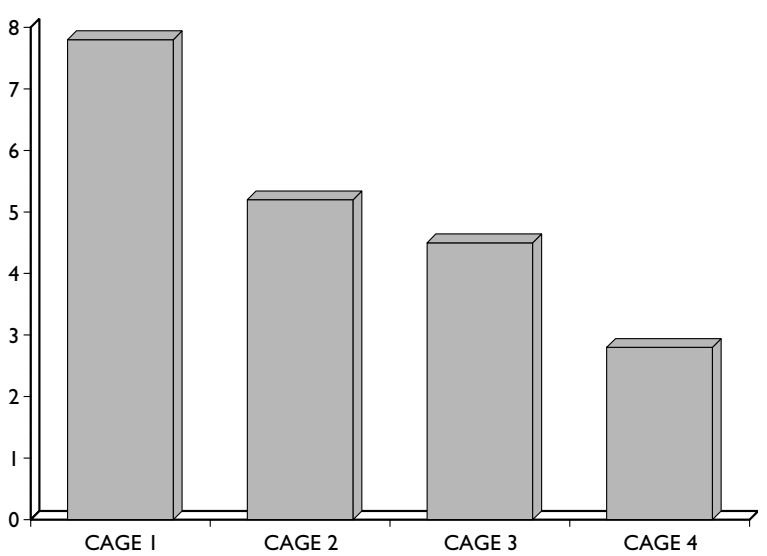

Figura 2. Prevalencia de alcoholismo en adultos meXiCANOS MAYORES DE 65 AÑOS SEGÚN LA ESCALA DE CAGE con su consumo por el anciano. Fink y colaboradores ${ }^{24}$ reportaron alcoholismo en el anciano en 7\%, y de los casos que ingresaron en unidades psiquiátricas, $28 \%$ se debía a este síndrome. En el presente trabajo, la prevalencia de alcoholismo en el anciano fue de $2.8 \%$; pero deberá considerarse que hasta $17.5 \%$ de los sujetos reportó problemática relacionada con la ingesta de alcohol (según CAGE).

En Estados Unidos se estima que hasta 23.3\% de las personas adultas fuman; se estima que para el año 2030 habrá 10 millones de muertes anuales en todo el mundo por consumo de tabaco. ${ }^{20,21,24}$ Según el último reporte del Cirujano General de ese país, ${ }^{18} 10.5 \%$ de los hombres ancianos y $10.7 \%$ de las mujeres reportaron tabaquismo intenso. El sector salud mexicano gasta $30 \mathrm{mil} \mathrm{millones}$ de pesos en la atención de afecciones asociadas con el tabaquismo; en el país se estima que hay 16 millones de mexicanos adictos a la nicotina, y su impacto en la población anciana se desconoce, el cual podría verse influenciado por la mortalidad prematura que ocasiona su consumo. En este análisis, la prevalencia de consumo severo y actual de tabaco fue de 14.3 por ciento.

El consumo y abuso de sustancias en la población anciana y su influencia en el deterioro cognoscitivo es un tema aún controvertido, ya que los diseños y metodología de los diferentes estudios que proponen considerar el alcohol y tabaco como factores de riesgo para deterioro cognoscitivo varían notablemente en los instrumentos de diagnóstico tanto para síndrome de dependencia al alcohol y tabaco como para diagnóstico

Cuadro III

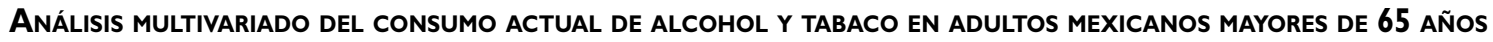

\begin{tabular}{|c|c|c|c|c|c|c|}
\hline \multirow[b]{2}{*}{ Variable } & \multicolumn{3}{|c|}{$\begin{array}{c}\text { Consumo de alcohol actual } \\
\qquad(n=|\quad| 40)\end{array}$} & \multicolumn{3}{|c|}{$\begin{array}{l}\text { Consumo de tabaco actual } \\
\qquad(n=668)\end{array}$} \\
\hline & $\overline{R R}$ & IC (95\%) & $p$ & $R R$ & IC (95\%) & $p$ \\
\hline Edad (a más edad) & - & - & $<0.001$ & - & - & 0.08 \\
\hline Sexo (masculino) & 3.17 & $2.6-3.8$ & $<0.001$ & 1.04 & $0.8-1.3$ & 0.73 \\
\hline Estado civil (casado [a]) & 0.89 & $0.7-1.0$ & 0.24 & - & - & NS \\
\hline Situación laboral (desempleado [a]) & 1.05 & $0.8-1.3$ & 0.65 & 1.21 & $0.9-1.5$ & 0.08 \\
\hline Escolaridad (a más años de estudios) & - & - & $<0.001$ & - & - & NS \\
\hline \multicolumn{7}{|l|}{ Funcionalidad (dependiente) } \\
\hline Básica & 0.79 & $0.6-0.9$ & 0.34 & 0.76 & $0.5-1.0$ & 0.05 \\
\hline Instrumentada & 0.70 & $0.5-0.9$ & 0.01 & 0.78 & $0.5-1.1$ & 0.18 \\
\hline Depresión & 0.80 & $0.6-0.9$ & 0.01 & - & - & NS \\
\hline
\end{tabular}

NS: no significativo (en análisis univariado) 


\section{Cuadro IV}

SubanÁlisis del CONSUMO de ALCOHOL y tABACO CON DETERIORO COGNOSCITIVo EN AdULTOS MEXICANOS MAYORES DE 65 AÑOS

\begin{tabular}{|c|c|c|c|}
\hline Variable & Número de ancianos & $\chi^{2}$ & $p$ \\
\hline Antecedentes de consumo de alcohol $(n=\mid 688)$ & 90 & 2.92 & 0.87 \\
\hline Ingesta actual de alcohol $(n=|| 40)$ & 50 & 6.59 & 0.01 \\
\hline \multicolumn{4}{|c|}{ Número de días de ingesta de alcohol a la semana $(n=1 \mid 140)$} \\
\hline Menos o igual a I & 31 & 7.00 & 0.03 \\
\hline $2-4$ & 7 & & \\
\hline $5-7$ & II & & \\
\hline Antecedentes de consumo de tabaco $(n=2253)$ & 121 & 2.13 & 0.14 \\
\hline Consumo actual de tabaco $(n=668)$ & 34 & 0.10 & 0.74 \\
\hline
\end{tabular}

de deterioro cognoscitivo, lo que limita obtener conclusiones definitivas. ${ }^{24-26}$

Autores de otros países que exploran este mismo fenómeno buscaron la relación entre fumar e ingesta fuerte de alcohol como factores de riesgo para deterioro cognoscitivo y deterioro funcional y encontraron una asociación estadística significativa. En México, estos hallazgos han sido poco reconocidos. Este análisis refuerza la percepción del riesgo a padecer deterioro cognoscitivo en aquellos sujetos que consumen alcohol hasta llegar a la adultez. Los factores asociados con el mayor consumo de alcohol serían pertenecer al género masculino, estar desempleado y con independencia funcional. En consecuencia, la mayor aportación de este estudio radica en la apertura de una línea de investigación hasta ahora poco explorada en México que intenta, a partir de las estimaciones obtenidas, generar conocimiento sobre la extensión y dinámica del alcoholismo y del tabaquismo como un problema más de salud pública con gran impacto, principalmente por las consecuencias sociales, funcionales y ahora cognoscitivas en el anciano mexicano. ${ }^{27-29}$

Una de las limitaciones de este estudio es ser retrospectivo; además, existen problemas metodológicos serios en la obtención de una muestra longitudinal confiable que pueda afirmar que el comportamiento cognoscitivo sea asociado directamente al consumo de estas sustancias, aunque es bien reconocido el impacto en las condiciones generales de salud y calidad de vida de los sujetos alcohólicos o con consumo de tabaco. Otro factor importante a explorar de manera más profunda es la relación entre el consumo de sustancias y la depresión, problemática frecuente en el anciano, que se ha asociado a mayor consumo de alcohol y tabaco. ${ }^{30}$
Las variables sociodemográficas más importantes asociadas con el consumo y abuso de sustancias fueron, en primer lugar, el género masculino, por ser hombres los que reportaron el mayor consumo de sustancias; el nivel socioeconómico alto, y la independencia funcional, lo que podría reflejar que, hasta ahora, la función social juega un factor importante en el consumo de sustancias. Sin embargo, es importante considerar que, a partir de los cambios demográficos que está sufriendo nuestro país, donde el mayor porcentaje de ancianos pertenece al género femenino, también es frecuente el consumo de sustancias, lo cual no se reporta ni explora por los médicos. Hacer un tamizaje de rutina con cualquiera de los instrumentos mencionados en este estudio podría ser un buen inicio para detectar y tratar la problemática del consumo y abuso de estas sustancias en la población anciana. ${ }^{31-34}$

\section{Agradecimientos}

Al doctor Luis Miguel Gutiérrez Robledo, jefe de la clínica de Geriatría del Instituto Nacional de Ciencias Médicas y Nutrición Salvador Zubirán, por la revisión crítica de la propuesta para el estudio, su apoyo incondicional y fomento a la investigación en el área de geriatría.

A la doctora Silvia Mejía, investigadora de la clínica de Geriatría del Instituto Nacional de Ciencias Médicas y Nutrición Salvador Zubirán, quien colaboró en este proyecto al compartir el análisis de datos de los sujetos con deterioro cognoscitivo. 


\section{Referencias}

I. López-lbor-Aliño J,Valdés-Miyar M. Manual diagnóstico y estadístico de los trastornos mentales. Texto revisado. 4a. ed. Madrid: Masson, 2002:45-50. 2. Ewing JA. Detecting alcoholism. The CAGE Questionnaire. JAMA 1984;252:1905-1907.

3. Moore AA, SeemanT, Morgenstern H, Beck JC, Reuben DB. Are there differences between older persons who screen positive on the CAGE questionnaire and the Short Michigan Alcoholism Screening test-geriatric version? J Am Geriatr Soc 2002;50:858-862.

4. Fingerhood M. Substance abuse in older people.J Am Geriatr Soc 2000;48:985-995.

5. Pirzada-Sattar S, Petty F, Burke W. Diagnosis and treatment of alcohol dependence in older alcoholics. Clin Geriatr Med 2003;19:4-17. 6. McGrath PJ, Nunes EV, Quitkin FM. Current concepts in the treatment of depression in alcohol-dependent patients. Psychiatr Clin N Am 2000;23:695-7II

7. Oslin DW, Pettinati HM.Volpicelli JR.Alcoholism treatment adherence: older age predicts better adherence and drinking outcomes. Am J Geriatr Psychiatry 2002;10:740-747.

8. Oslin DW, Cary MS. Alcohol-related dementia: validation of diagnostic criteria.Am J Geriatr Psychiatry 2003; I (4):44I-447.

9. Herbert LE, Scherr PA, Beckett LA. Relation of smoking and lowto moderate alcohol consumption to change in cognitive function: a longitudinal study in a defined community of persons. Am J Epidemiol 1993;137:881-89|.

10. Hajat S, Haines A, Bulpitt C, Fletcher A. Patterns and determinants of alcohol consumption in people aged 75 years and older: results from the-MRC trial of assessment and management of older people in the community.Age Ageing 2004;33:170- 177.

II. Mersy DJ. Recognition of alcohol and substance abuse.Am Fam Physician 2003;67:1529-1532,1535-1536.

12. Zisserson RN, Oslin DW.Alcoholism and at-risk drinking in the older population. Geriatrics Times 2003;5:5.

13. Uchimoto S. Tsumura K, Hayashi T, et al. Impact of cigarrete smoking on the incidence of Type 2 diabetes mellitus in middle-aged Japanese men: the Osaka Health Survey. Diabet Med 1999;16:951-955.

14. Unverzagt FW, Gao S, Baiyewu. Prevalence of cognitive impairment: data from the Indianapolis study of health and aging. Neurology 2001;57:123-127.

15. Rigler SK.Alcoholism in the elderly.Am Fam Physician 2000;61: $1710-1716$.
16. Orgogozo JM, Dartigues JF, Lafont S, et al.Wine consumption and dementia in the elderly: a prospective community study in the Bordeaux area. Rev Neurol 1997;153:185-192.

17. Cervilla JA, Prince M, Mann A. Smoking, drinking and incident cognitive impairment: a cohort community included in the Gospel Oak project.J Neurol Neurosurg Psychiatry 2000;68:622-626.

18. Camacho TC, Kaplan GA, Cohen RD. Alcohol consumption and mortality in Alameda County.J Chron Dis 1987;40:229-236.

19. Ott A,Andersen K, Dewey ME, Letenneur L, Brayne C, Copeland JR, et al. Effect of smoking on global cognitive function in nondemented elderly. Neurology 2004;62:920-924.

20. Merchant C, Tang MX,Albert S, Manly J, Stern Y, Mayeux R. The influence of smoking on the risk of Alzheimer's disease. Neurology 1999;52:1408-1412.

2I. Ford AB, Mefrouche Z, Friedland RP, Debanne SM. Smoking and cognitive impairment: a population-based study. J Am Geriatr Soc 1996;44:905-909.

22. Letenneur L, Dartigues JF, Commenges D, Barbeger-Gateaus P, et al. Tobacco consumption and cognitive impairment in elderly people.A population-based study.Ann Epidemiol 1994;4:449-454.

23. Estudio Nacional de Salud y Envejecimiento en México. Disponible en: http://www.mhas.pop.upenn.edu/

24. Chun BY, Dobson AJ, Heller RF. Smoking and the incidence of coronary heart disease in an Australian population. Med J Aust 1993;159: 508-5 I2. 25. Letenneur L, Dartigues JF, Commenges D, Barberger-Gateau P, Tessier JF, Orgogozo JM. Tobacco consumption and cognitive impairment in elderly people.A population-based study. Ann Epidemiol 1994;4:449-454. 26. Mejía S, Miguel A, Gutiérrez LM,Villa A, Ostrosky-Solís F. Comparative analysis of cognitive impairment among Mexicans and Spanish-speaking immigrants elders. Internat Psychogeriatr 2004 (en prensa).

27. GregW. Alcohol: a much neglected risk factor in elderly mental disorders. Curr Opin Psychiatry 2003;16:609-614. 28. Dorozynki A. Wine may prevent dementia. Br Med J 1997;3 14:997. 29. Tyas SL, Koval JJ, Pederson LL. Does an interaction between smoking and drinking influence the risk of Alzheimer's disease? Results from three Canadian data sets. Stat Med 2000;19:1685-1696.

30. Appel DW, Aldrich TK. Smoking cessation in the elderly. Clin Geriatr Med 2003;19:1-15

31. Meng S, Tang Z, Biao C.A longitudinal study of factors influencing cognitive impairment of aged people. Clin Geriatr Med 2000;19:2 II-2 I4. 32.Vilk PW, Cellucci T, Jarchow A, Hedt J. Cognitive impairment in substance abuse. Psychiatr Clin North Am 2004;27:I-5.

33. Breteler MM, Bots ML, Ott A, Shinton R, Beevers G. Risk factors for vascular disease and dementia. Haemostasis 1998;22:621-623.

34. Kelton MC, Kahn HJ, Conrath CL, et al. The effects of nicotine on Parkinson's disease. Brain Cogn 2000;43:274-282. 